\title{
FDA Individual Case Safety Report Terminology
}

National Cancer Institute

\section{Source}

National Cancer Institute. FDA Individual Case Safety Report Terminology. NCI

Thesaurus. Code C54447.

Terminology used in individual case reports for voluntary and mandatory reporting of adverse reactions or adverse events during both pre and post approval periods of medical product development. 